\title{
Changes among male and female visitors to practitioners of complementary and alternative medicine in a large adult Norwegian population from 1997 to 2008 (The HUNT studies)
}

\author{
Aslak Steinsbekk*, Marit B Rise and Roar Johnsen
}

\begin{abstract}
Background: The aim was to investigate changes in the prevalence and characteristics of male and female visitors to practitioners of complementary and alternative medicine (CAM) in a large adult population from 1997 to 2008.

Methods: Two cross sectional adult total population health surveys from Central Norwegian (the Nord-Trøndelag Health Studies (HUNT)). In 1997 42,277 and in 2008 50,713 respondents were included. Variables included demographics (age, education, working status), lifestyle (daily smoker, did hard physical activities), health status (self-rated health status, recent complaints, chronic complaints, psychiatric complaints, a range of diseases) and health care use (visit general practitioner, chiropractor). A test of difference between the results of multivariable logistic regression models for each year, including all variables, was used to analyse changes from 1997 to 2008.

Results: In 1997 9.4\% (95\%Cl 9.1-9.6) of the population had visited a CAM practitioner in the last 12 months and this increased to $12.6 \%(12.3-12.9)$ in 2008 ( $p<0.001$ for difference). Prevalence of CAM use in females was almost twice as high as that in males both years. For males, the significant changes from 1997 to $2008(p<0.05)$ were an increase in odds of visiting for those under 50 years, who had a recent complaint, were widower or did hard physical activities. There was a decrease for males who had a university degree, psychiatric complaint or hay fever. For females there was an increase in the odds for those under 50 years, who had a recent complaint or chronic complaint. It was a decrease for females with reported fair global health, psychiatric complaint, hay fever or if they had visited a chiropractor.
\end{abstract}

Conclusion: The increase in visits was mainly among younger people of both genders with more limited complaints. A larger proportion of the more healthy part of the population is increasing their visits to CAM practitioners.

Keywords: Complementary Therapies, Norway, Utilisation, Population

\section{Background}

Complementary and Alternative Medicine (CAM) are treatment modalities outside the dominant health care system. Several studies have investigated the reasons why people use these practices [1]. Issues such as control and participation, perceptions of illness, holism and natural treatments, and general philosophies of life have been found to be related to CAM use.

\footnotetext{
* Correspondence: aslak.steinsbekk@ntnu.no

Norwegian University of Science and Technology (NTNU), Department of Public Health and General Practice, Trondheim, Norway
}

There are a number of studies investigating the characteristics of CAM users. Based on data from larger cross sectional surveys, being middle aged, female and having higher education is often associated with CAM use [2]. However, there are several types of CAM use; visits to practitioners like homeopaths and acupuncturists, performing CAM self practices like yoga and self treatment with CAM products like herbs [3].

To distinguish between the different types of CAM use is intuitively an important step since those visiting practitioners could differ from those using self treatment. There are some studies that have either focused 
on CAM visitors or identified these among all CAM users [4-9]. Another development is to investigate changes over time. There are to date only few studies that have analysed whether prevalence and characteristics change over time [6,10-14]. In USA there was an increase in total CAM use in the 1990's [13] and this has been stable on a high level in the 2000's [11,12], a trend comparable to Australia for total CAM use although there has been an increase in visitors to CAM practitioners [6]. In smaller studies in Israel, there has been a steady increase in the proportion of adults visiting a CAM practitioner from 1993 to 2007 [7] and also Ireland saw an increase in visits from 1998 to 2002 [10].

To further expand on this research, the aim was to investigate changes from 1997 to 2008 in the prevalence and characteristics of male and female visitors to practitioners of complementary and alternative medicine (CAM) in a large adult population in Norway.

\section{Methods}

The data are from two cross sectional total population studies conducted in one county in Central Norway, the second (HUNT 2, conducted 1995-97) and third (HUNT 3, conducted 2006-08) Nord-Trøndelag Health Study (http://www.medisin.ntnu.no/hunt/). The county and its population is considered fairly representative of Norway concerning geographical, demographic and occupational structure [15], but have no larger cities and the population has an income and education level slightly below the national average. For both surveys, all residents aged 20 years or over were invited to participate by post and received the first questionnaire $(\mathrm{Q} 1)$ attached to the invitation. This was to be returned at a screening station where a brief medical examination was conducted and a second questionnaire $(\mathrm{Q} 2)$ to be returned by post was handed out. The HUNT studies have been approved by the Regional Committee for Medical and Health Research Ethics, Central Norway and the Norwegian Data Inspectorate.

In HUNT 2, 92,936 inhabitants were invited and a total of 65,495 (70.5\%) persons participated. Of these, 41,734 (63.7\%) participants answered a question on CAM use which was in Q2 and were included in the present study. In HUNT 3, 94,194 were invited, 50,827 participated (54.0\%) and $50713(99.8 \%)$ answered a question on CAM use in Q1.

The total population in Norway is nearly 5 million and it is among the countries that have highest total expenditure on health per capita. The Norwegian health care system includes provision of health care services for all citizens based on need regardless of personal income. CAM practitioners operate outside the government-funded health care system and everyone can call themselves a CAM practitioner and treat patients.

\section{CAM visitor}

A CAM visitor was defined as a participant who had consulted one or more CAM practitioners by answering yes to the question: "During the last 12 months, have you visited homeopath, acupuncturist, reflexologist, layer on of hands or another alternative treatment practitioner? (Yes/No)". There was a separate question about visits to chiropractors immediately before the CAM question, but as they are authorised health personnel in Norway, visit to a chiropractor was not included in CAM visits.

\section{Demographics}

The participants' gender, age, marital status and education level were taken from public registers and a question on cohabitation. Level of education was reclassified as compulsory school, middle level education (including vocational education below university level) and university degree. Currently working were those answering yes to being in paid employment.

\section{Lifestyle}

Participants were classified as smokers or non-smokers based on whether or not they were daily smokers of cigarettes, cigar and/or pipe. Activity level was dichotomised to doing more or less than 3 hours of hard physical activity weekly during spare time last year.

\section{Health status}

Several measures of self reported health status were used (question with answering categories):

1. Global health: How is your health at the moment? (poor, fair, good, very good).

2. Anxiety and depression: The Hospital Anxiety and Depression Scale (HADS-T) was used (14 items, score ranging from 0 to 42 , the cut off point for the detection of any mental disorder is found to be 17 [16].

3. Recent complaint (yes to one or more of these questions):

- Have you suffered from Nausea/Heartburn/Diarrhoea/Constipation/Breathlessness in the last 12 months? $($ Never $=$ No $/$ Sometimes $=$ Yes $/$ Often $=$ Yes $)$.

- Have you experienced any stiffness or pain in your muscles or joints that has lasted for more than three consecutive months during the last year? (Yes/No).

- Have you suffered from headache in the last 12 months (Yes/No).

4. Chronic complaint: Do you suffer from any long standing (for at least one year) limiting somatic or psychiatric illness, disease or disability? (Yes/No).

5. Psychiatric complaint: Do you have or have you had psychiatric complaints that you have sought help for? (Yes/No).

6.- 8. Asthma, Diabetes, or Cancer: Do you have or have you had asthma/diabetes/cancer? (Yes/No). 
9. Hay fever: Do you have hay fever? (Yes/no).

10. Cardiovascular disease (yes to one or more of these questions): Do you have or have you had Acute myocardial infarction/Angina pectoris/Stroke? (Yes/No).

11. Musculoskeletal disease (yes to one or more of these questions): Have you been diagnosed with Osteoporosis/Fibromyalgia/Arthritis/Artroses/Bechterew/ Other longstanding musculoskeletal disease? (Yes/No).

\section{Health care utilisation}

Health care utilisation was answering yes to questions about visits the last 12 months:

- Physician: During the past 12 months, have you visited a General practitioner/Specialist outside hospital/ Psychiatric specialist in hospital/Somatic specialist in hospital? (Yes/No).

- Chiropractor: During the last 12 months, have you visited a chiropractor? (Yes/No).

\section{Statistical Analyses}

Pearson chi-square tests were used to compare users with non-users for each year separately. Spearman's rho was used to check for co-linearity and the correlation coefficient was highest both years for currently working/age (1997; 0.410, 2008; 0.561) and chronic complaint/global health $(1997 ; 0.409,2008 ; 0.507)$. The multivariable analysis (adjusted odds ratio - adjOR) was undertaken by logistic regression using models where all variables were included to identify associations between CAM use and the other variables also for both years. Due to the large size of the dataset and the number of comparisons, statistical significance was accepted at the $1 \%$ level $(\mathrm{p}<0.01)$ for these tests. To compare the findings of the bivariable and multivariable analysis for each year with each other, a test of difference for comparison of two independent studies was used [17]. For the multivariable analysis, the Ratio Odds Ratio (ROR) was calculated, which is the ration from a comparison of two odds ratios. A ROR higher than 1 indicates higher odds of visiting a CAM practitioner in 2008 than in 1997. Statistical significance accepted at the $5 \%$ level $(\mathrm{p}<0.05)$ for these tests. The adjOR and ROR are given with $95 \%$ confidence intervals (95\%CI). All data were analysed using SPSS statistics version 17.0.0 released Aug 23. 2008 (SPSS Inc, Chicago, USA).

\section{Results}

The final data from 1997 comprised 19,490 males and 22,244 females and 22,998 males and 27,715 females were included in 2008 .

In total $9.4 \%$ (95\% CI 9.1-9.6, females $12.2 \%$, males $6.1 \%$ ) of the population visited a CAM practitioner in 1997 and this increased significantly ( $\mathrm{p}<0.001$ ) to $12.6 \%$ (95\%CI $12.3-12.9$, females $16.2 \%$, males $8.3 \%$ ) in 2008 (table 1). Thus, nearly twice as many females had visited a CAM practitioner both in 1997 and 2008 compared to males. There was also a significant $(\mathrm{p}<0.001)$ increase in CAM visits for both genders (males $2.2 \%$ points, females $3.9 \%$ points) from 1997 to 2008 .

The bivariate analysis showed that for both males and females, having poorer self reported global health, higher anxiety and depression score (HADS-T), chronic or psychiatric complaint and having visited a chiropractor were the variables most strongly associated with visit to a CAM practitioner (table 2). In 2008, compared to 1997 , there was a significant increase in the proportion of visitors for most of the variables. For both males and females, the most striking changes beyond the general increase were an increase in the younger age groups and among those who did hard physical activity.

\section{CAM visit males}

The multivariable logistic regression showed that for males in 1997, age had no influence (table 3). In 2008 ( $\mathrm{p}<0.01$ ) being above 50 years old (adjusted Odds Ratio (adjOR) from 0.7 for those 50-59 years going down to 0.4 for those 70 years and older) was associated with decreased odds of visiting a CAM practitioner and this was significantly lower than in 1997 (Ratio Odds Ratio (ROR) 0.4 for those 50-59 years to 0.2 for those 80 years and older).. Having poorer self reported global health was associated with increased odds of visiting a CAM practitioner both in 1997 (adjOR from 1.4 for good health increasing to 3.8 for poor health) and in 2008 (adjOR from 1.3 for good health increasing to 2.9 for poor health), and although the odds was lower in 2008, it was not statistically significantly different from 1997 . Visit to a physician (adjOR 2.0) or a chiropractor (adjOR 3.2) was also associated with CAM visits in 1997 and 2008 (physician adjOR 1.8, chiropractor adjOR 2.6), but there were no statistical significant change during these eleven years.

Table 1 Prevalence of visits to complementary and alternative medicine (CAM) practitioners during the last 12 months in 1997 and 2008 for males and females

\begin{tabular}{|c|c|c|c|}
\hline Visited CAM practitioner in & Total & Male & Female \\
\hline 1997 & $\begin{array}{l}(N=41734) \\
9.4 \%(9.1-9.6)\end{array}$ & $\begin{array}{l}(N=19490) \\
6.1 \%(5.8-6.4)\end{array}$ & $\begin{array}{c}(\mathrm{N}=22244) \\
12.2 \%(11.8-12.7)\end{array}$ \\
\hline 2008 & $\begin{array}{c}(\mathrm{N}=50713) \\
12.6 \%(12.3-12.9)\end{array}$ & $\begin{array}{l}(\mathrm{N}=22998) \\
8.3 \%(7.9-8.6)\end{array}$ & $\begin{array}{c}(\mathrm{N}=27715) \\
16.2 \%(15.7-16.6)\end{array}$ \\
\hline
\end{tabular}


Table 2 Bivariate analysis with percentage of visits to CAM practitioners (\%CAM) in 1997 and 2008 for males and females ( $N$ given in table)

\begin{tabular}{|c|c|c|c|c|c|c|c|c|c|c|c|c|}
\hline & \multicolumn{6}{|c|}{ Male } & \multicolumn{6}{|c|}{ Female } \\
\hline & \multicolumn{2}{|c|}{2008} & \multicolumn{2}{|c|}{1997} & \multicolumn{2}{|c|}{2008 vs 1997} & \multicolumn{2}{|c|}{2008} & \multicolumn{2}{|c|}{1997} & \multicolumn{2}{|c|}{2008 vs 1997} \\
\hline & $\mathrm{N}$ & $\%$ CAM & $\mathrm{N}$ & $\%$ CAM & Diff & P-value & $\mathrm{N}$ & $\%$ CAM & $\mathrm{N}$ & $\%$ CAM & Diff & P-value \\
\hline Visited a CAM practitioner & 22998 & $8.3 \%^{*}$ & 19490 & $6.1 \% *$ & $2.2 \% *$ & $<0.001$ & 27715 & $16.2 \% *$ & 22244 & $12.2 \%^{*}$ & $3.9 \%^{*}$ & $<0.001$ \\
\hline $\begin{array}{l}\text { Age group } \\
\text { - Under } 30\end{array}$ & 1852 & $7.9 \% *$ & 2766 & $4.0 \% *$ & $4.0 \%^{*}$ & $<0.001$ & 2634 & $13.7 \% *$ & 3546 & $9.2 \% *$ & $4.6 \% *$ & $<0.001$ \\
\hline$-30-39$ & 2843 & $9.4 \% *$ & 3738 & $5.6 \% *$ & $3.8 \%^{*}$ & $<0.001$ & 3998 & $18.1 \% *$ & 4606 & $12.1 \% *$ & $5.9 \%^{*}$ & $<0.001$ \\
\hline$-40-49$ & 4546 & $9.7 \% *$ & 4347 & $5.9 \% *$ & $3.8 \% *$ & $<0.001$ & 5434 & $18.5 \% *$ & 4898 & $13.0 \% *$ & $5.6 \% *$ & $<0.001$ \\
\hline$-50-59$ & 5400 & $8.8 \% *$ & 3476 & $6.3 \% *$ & $2.5 \%^{*}$ & $<0.001$ & 5989 & $18.3 \% *$ & 3701 & $14.9 \%^{*}$ & $3.4 \% *$ & $<0.001$ \\
\hline$-60-69$ & 4650 & $7.4 \% *$ & 2431 & $7.5 \%{ }^{*}$ & $-0.1 \%$ & 0.892 & 5106 & $14.8 \% *$ & 2560 & $13.5 \%^{*}$ & $1.3 \%$ & 0.117 \\
\hline - 70-79 & 2649 & $6.3 \%^{*}$ & 2146 & $7.2 \% *$ & $-1.0 \%$ & 0.188 & 3076 & $12.6 \% *$ & 2148 & $10.6 \% *$ & $2.0 \%^{*}$ & 0.025 \\
\hline - Over 80 & 980 & $5.6 \% *$ & 586 & $9.7 \% *$ & $-4.1 \% *$ & 0.002 & 1417 & $9.2 \% *$ & 785 & $9.8 \%{ }^{*}$ & $-0.6 \%$ & 0.625 \\
\hline $\begin{array}{l}\text { Education } \\
\text { - Compulsory school }\end{array}$ & 4458 & $7.6 \% *$ & 5435 & $6.3 \%$ & $1.3 \% *$ & 0.009 & 6166 & $14.1 \% *$ & 7436 & $12.3 \%$ & $1.8 \%^{*}$ & 0.002 \\
\hline - Middle level & 13200 & $8.9 \% *$ & 9336 & $6.0 \%$ & $3.0 \% *$ & $<0.001$ & 13216 & $17.6 \% *$ & 9058 & $12.7 \%$ & $4.9 \%^{*}$ & $<0.001$ \\
\hline - University & 5067 & $7.0 \% *$ & 4115 & $5.8 \%$ & $1.2 \% *$ & 0.016 & 8006 & $15.5 \% *$ & 4886 & $11.3 \%$ & $4.2 \% *$ & $<0.001$ \\
\hline $\begin{array}{l}\text { Marital status } \\
\text { - Married/cohabiting }\end{array}$ & 17380 & $8.5 \%$ & 15186 & $6.2 \% *$ & $2.3 \% *$ & $<0.001$ & 20123 & $16.7 \% *$ & 17107 & $12.5 \% *$ & $4.2 \%{ }^{*}$ & $<0.001$ \\
\hline - Single & 4017 & $7.5 \%$ & 3142 & $4.7 \% *$ & $2.8 \% *$ & $<0.001$ & 3465 & $15.2 \% *$ & 2125 & $10.5 \%^{*}$ & $4.7 \% \%^{*}$ & $<0.001$ \\
\hline - Divorced/separated & 1069 & $8.1 \%$ & 615 & $9.4 \% *$ & $-1.3 \%$ & 0.363 & 1603 & $18.3 \%^{*}$ & 878 & $15.3 \%^{*}$ & $3.0 \%$ & 0.057 \\
\hline - Widow(er) & 461 & $8.7 \%$ & 512 & $8.0 \% *$ & $0.7 \%$ & 0.706 & 2412 & $11.9 \%^{*}$ & 2072 & $10.6 \%^{*}$ & $1.4 \%$ & 0.148 \\
\hline Currently working & 15268 & $8.6 \%$ & 13914 & $5.5 \% *$ & $3.0 \%{ }^{*}$ & $<0.001$ & 17090 & $17.4 \%^{*}$ & 14037 & $12.3 \%$ & $5.1 \% *$ & $<0.001$ \\
\hline $\begin{array}{l}\text { Current lifestyle: } \\
\text { - Daily smoker }\end{array}$ & 3919 & $7.1 \% *$ & 5295 & $5.1 \% *$ & $2.0 \% *$ & $<0.001$ & 5565 & $16.0 \%$ & 6361 & $11.5 \%$ & $4.5 \% *$ & $<0.001$ \\
\hline - Hard physical activity & 3163 & $9.8 \% *$ & 2568 & $5.1 \%$ & $4.7 \%^{*}$ & $<0.001$ & 2936 & $17.7 \%$ & 1287 & $12.0 \%$ & $5.7 \% *$ & $<0.001$ \\
\hline $\begin{array}{l}\text { Global Health: } \\
\text { - Very good }\end{array}$ & 3527 & $5.4 \% *$ & 3399 & $3.1 \% *$ & $2.3 \%{ }^{*}$ & $<0.001$ & 4172 & $10.3 \% *$ & 3864 & $6.4 \%^{*}$ & $3.9 \% *$ & $<0.001$ \\
\hline - Good & 13475 & $7.4 \% *$ & 11763 & $5.0 \% *$ & $2.3 \%^{*}$ & $<0.001$ & 14787 & $15.2 \%^{*}$ & 12524 & $10.4 \%^{*}$ & $4.8 \% *$ & $<0.001$ \\
\hline - Fair & 4874 & $12.5 \% *$ & 3934 & $10.8 \% *$ & $1.6 \% *$ & 0.017 & 7142 & $21.3 \%^{*}$ & 5343 & $19.8 \%^{*}$ & $1.5 \% *$ & 0.035 \\
\hline - Poor & 314 & $17.2 \% *$ & 294 & $18.7 \%^{*}$ & $-1.5 \%$ & 0.628 & 389 & $27.8 \%^{*}$ & 332 & $26.5 \%^{*}$ & $1.3 \%$ & 0.705 \\
\hline $\begin{array}{l}\text { Anxiety and depression } \\
-0-4\end{array}$ & 6196 & $7.6 \% *$ & 6735 & $4.9 \% *$ & $2.7 \% *$ & $<0.001$ & 7714 & $13.9 \% *$ & 7561 & $8.8 \%^{*}$ & $5.1 \% *$ & $<0.001$ \\
\hline$-5-9$ & 6218 & $8.1 \% *$ & 6998 & $5.3 \% *$ & $2.8 \%^{*}$ & $<0.001$ & 7351 & $16.5 \% *$ & 7538 & $11.7 \%^{*}$ & $4.8 \% *$ & $<0.001$ \\
\hline$-10-14$ & 3066 & $9.3 \% *$ & 3481 & $7.4 \% *$ & $1.9 \%^{*}$ & 0.005 & 3930 & $18.9 \%^{*}$ & 3881 & $15.0 \% *$ & $4.0 \%^{*}$ & $<0.001$ \\
\hline$-15-19$ & 1123 & $11.6 \% *$ & 1206 & $9.5 \% *$ & $2.0 \%$ & 0.109 & 1719 & $22.4 \%^{*}$ & 1646 & $19.0 \% *$ & $3.4 \% *$ & 0.014 \\
\hline - 20 and higher & 453 & $11.7 \% *$ & 558 & $13.3 \%^{*}$ & $-1.6 \%$ & 0.456 & 811 & $22.3 \% *$ & 872 & $21.1 \% *$ & $1.2 \%$ & 0.545 \\
\hline Recent complaint & 15562 & $9.3 \% *$ & 12053 & $6.5 \% *$ & $2.8 \%^{*}$ & $<0.001$ & 20995 & $17.6 \% *$ & 14642 & $12.9 \% *$ & $4.7 \% *$ & $<0.001$ \\
\hline Chronic complaint & 7096 & $12.1 \% *$ & 4366 & $10.5 \% *$ & $1.6 \% *$ & 0.009 & 8821 & $22.2 \% *$ & 4608 & $19.0 \% *$ & $3.2 \% *$ & $<0.001$ \\
\hline Psychiatric complaint & 2178 & $12.3 \%^{*}$ & 1511 & $13.6 \% *$ & $-1.3 \%$ & 0.259 & 4438 & $21.9 \% *$ & 3025 & $20.2 \%^{*}$ & $1.7 \%$ & 0.070 \\
\hline $\begin{array}{l}\text { Diseases } \\
\text { - Asthma }\end{array}$ & 2016 & $9.5 \%$ & 1662 & $8.0 \% *$ & $1.5 \%$ & 0.117 & 2706 & $19.0 \% *$ & 1840 & $16.7 \%^{*}$ & $2.3 \% *$ & 0.044 \\
\hline - Hay fever & 4185 & $9.4 \% *$ & 3197 & $8.1 \% *$ & $1.3 \%$ & 0.051 & 6185 & $19.3 \% *$ & 4405 & $16.3 \%^{*}$ & $3.1 \% *$ & $<0.001$ \\
\hline - Heart disease & 3251 & $7.4 \%$ & 1568 & $7.6 \% *$ & $-0.2 \%$ & 0.827 & 2212 & $14.0 \% *$ & 972 & $13.7 \%$ & $0.3 \%$ & 0.830 \\
\hline - Cancer & 1116 & $8.5 \%$ & 558 & $10.0 \% *$ & $-1.5 \%$ & 0.305 & 1541 & $15.5 \%$ & 833 & $13.9 \%$ & $1.6 \%$ & 0.302 \\
\hline - Diabetes & 1136 & $8.3 \%$ & 499 & $7.0 \%$ & $1.3 \%$ & 0.384 & 1047 & $13.9 \%$ & 497 & $12.5 \%$ & $1.5 \%$ & 0.429 \\
\hline - Musculoskeletal disease & 3088 & $11.2 \%^{*}$ & 1904 & $9.3 \% *$ & $1.9 \%^{*}$ & 0.032 & 7369 & $19.0 \% *$ & 3259 & $17.8 \%^{*}$ & $1.2 \%$ & 0.148 \\
\hline $\begin{array}{l}\text { Visited last year: } \\
\text { - Physician }\end{array}$ & 17769 & $9.5 \% *$ & 11738 & $8.1 \% *$ & $1.4 \%^{*}$ & $<0.001$ & 23907 & $17.2 \%^{*}$ & 16787 & $14.2 \%^{*}$ & $3.1 \% *$ & $<0.001$ \\
\hline - Chiropractor & 1980 & $19.3 \% *$ & 793 & $19.2 \% *$ & $0.1 \%$ & 0.940 & 2016 & $29.3 \% *$ & 649 & $34.4 \% *$ & $-5.1 \% *$ & 0.014 \\
\hline
\end{tabular}

Test of proportions is used to compare changes from 1997 to 2008 with a positive difference (Diff) indicating higher prevalence in 2008.

* p-value $<0.01$ for each year and $p$-value $<0.05$ for comparison 
Table 3 Adjusted odds ratio (adjOR) with 95\% confidence intervals $(95 \% \mathrm{Cl})$ from multivariate logistic regression models for visits to CAM practitioners for males in 2008 ( $N=16311$ ) and in $1997(N=16488)$, for females in 2008 ( $N=20469)$ and in $1997(N=17587)$, and test of difference between the time points for males and females with Ratio Odds Ratio (ROR)

\begin{tabular}{|c|c|c|c|c|c|c|c|c|c|c|c|c|}
\hline & \multicolumn{6}{|c|}{ Male $^{a}$} & \multicolumn{6}{|c|}{ Female $^{a}$} \\
\hline & \multicolumn{2}{|c|}{2008} & \multicolumn{2}{|c|}{1997} & \multicolumn{2}{|c|}{2008 vs 1997} & \multicolumn{2}{|c|}{2008} & \multicolumn{2}{|c|}{1997} & \multicolumn{2}{|c|}{2008 vs 1997} \\
\hline & $\begin{array}{l}\text { AdjOR (95\% } \\
\mathrm{Cl})\end{array}$ & $\begin{array}{l}\text { P-value } \\
\text { 1) }\end{array}$ & $\begin{array}{l}\text { AdjOR }(95 \% \\
\mathrm{Cl})\end{array}$ & $\begin{array}{c}\text { P-value } \\
\text { 1) }\end{array}$ & $\begin{array}{l}\text { ROR }(95 \% \\
\text { Cl) }\end{array}$ & $\begin{array}{l}\text { P-value } \\
\text { 2) }\end{array}$ & $\begin{array}{l}\text { AdjOR }(95 \% \\
\mathrm{Cl})\end{array}$ & $\begin{array}{c}\text { P-value } \\
\text { 1) }\end{array}$ & $\begin{array}{l}\text { AdjOR (95\% } \\
\mathrm{Cl})\end{array}$ & $\begin{array}{l}\text { P-value } \\
\text { 1) }\end{array}$ & $\begin{array}{l}\text { ROR }(95 \% \\
\quad \mathrm{Cl})\end{array}$ & $\begin{array}{l}\text { P-value } \\
\text { 2) }\end{array}$ \\
\hline $\begin{array}{l}\text { Age group } \\
\text { - Under } 30\end{array}$ & Ref & & Ref & & & & Ref & & Ref & & & \\
\hline$-30-39$ & $1.0(0.7-1.3)$ & 0.912 & $1.2(0.9-1.5)$ & 0.204 & $0.8(0.6-1.2)$ & 0.359 & $1.2(1.0-1.4)$ & 0.068 & $1.3(1.1-1.5)^{*}$ & 0.003 & $0.9(0.7-1.2)$ & 0.515 \\
\hline$-40-49$ & $0.8(0.6-1.1)$ & 0.248 & $1.1(0.9-1.5)$ & 0.367 & $0.8(0.5-1.1)$ & 0.145 & $1.2(1.0-1.4)$ & 0.055 & $1.3(1.1-1.5)^{*}$ & 0.003 & $0.9(0.7-1.2)$ & 0.495 \\
\hline$-50-59$ & $0.7(0.5-0.9)^{*}$ & 0.003 & $1.1(0.8-1.4)$ & 0.695 & $0.6(0.4-0.9)^{*}$ & 0.017 & $1.0(0.9-1.2)$ & 0.655 & $1.3(1.1-1.6)^{*}$ & 0.001 & $0.8(0.6-1.0)^{*}$ & 0.044 \\
\hline$-60-69$ & $0.5(0.4-0.7)^{*}$ & $<0.001$ & $1.3(1.0-1.7)$ & 0.092 & $0.4(0.3-0.6)^{*}$ & $<0.001$ & $0.9(0.7-1.0)$ & 0.091 & $1.3(1.0-1.6)$ & 0.022 & $0.7(0.5-0.9)^{*}$ & 0.005 \\
\hline$-70-79$ & $0.4(0.3-0.6)^{*}$ & $<0.001$ & $1.2(0.9-1.7)$ & 0.224 & $0.4(0.2-0.6)^{*}$ & $<0.001$ & $0.8(0.6-1.0)$ & 0.040 & $1.0(0.8-1.3)$ & 0.980 & $0.8(0.6-1.1)$ & 0.167 \\
\hline - Over 80 & $0.4(0.2-0.6)^{*}$ & $<0.001$ & $1.5(1.0-2.4)$ & 0.063 & $0.2(0.1-0.5)^{*}$ & $<0.001$ & $0.5(0.4-0.7)^{*}$ & $<0.001$ & $0.9(0.7-1.3)$ & 0.696 & $0.6(0.4-0.9)^{*}$ & 0.026 \\
\hline $\begin{array}{l}\text { Education } \\
\text { - Compulsory school }\end{array}$ & Ref & & Ref & & & & Ref & & Ref & & & \\
\hline - Middle level & $1.0(0.9-1.2)$ & 0.778 & $1.2(1.0-1.4)$ & 0.065 & $0.9(0.7-1.1)$ & 0.263 & $1.2(1.1-1.4)^{*}$ & $<0.001$ & $1.1(1.0-1.3)$ & 0.023 & $1.1(0.9-1.2)$ & 0.401 \\
\hline - University & $0.8(0.7-1.0)$ & 0.040 & $1.1(0.9-1.4)$ & 0.154 & $0.7(0.5-0.9)^{*}$ & 0.014 & $1.1(0.9-1.2)$ & 0.320 & $1.0(0.9-1.2)$ & 0.656 & $1.0(0.9-1.2)$ & 0.740 \\
\hline $\begin{array}{l}\text { Marital status } \\
\text { - Married/cohabiting }\end{array}$ & Ref & & Ref & & & & Ref & & Ref & & & \\
\hline - Single & $0.7(0.6-0.9)^{*}$ & 0.001 & $0.9(0.7-1.1)$ & 0.274 & $0.8(0.6-1.1)$ & 0.110 & $1.0(0.8-1.1)$ & 0.642 & $1.0(0.8-1.2)$ & 0.829 & $1.0(0.8-1.2)$ & 0.884 \\
\hline - Divorced/separated & $0.9(0.7-1.3)$ & 0.684 & $1.4(1.0-1.9)$ & 0.033 & $0.7(0.4-1.0)$ & 0.075 & $1.0(0.9-1.2)$ & 0.807 & $1.1(0.9-1.3)$ & 0.551 & $1.0(0.7-1.3)$ & 0.756 \\
\hline - Widow(er) & $1.7(1.1-2.6)^{*}$ & 0.009 & $1.0(0.6-1.4)$ & 0.819 & $1.8(1.0-3.2)^{*}$ & 0.048 & $1.1(0.9-1.3)$ & 0.523 & $0.9(0.7-1.1)$ & 0.231 & $1.2(0.9-1.6)$ & 0.187 \\
\hline Currently working & $1.0(0.8-1.2)$ & 0.886 & $1.0(0.8-1.2)$ & 0.983 & $1.0(0.8-1.3)$ & 0.935 & $1.2(1.1-1.4)^{*}$ & $<0.001$ & $1.1(1.0-1.3)$ & 0.053 & $1.1(0.9-1.3)$ & 0.296 \\
\hline $\begin{array}{l}\text { Current lifestyle: } \\
\text { - Daily smoker }\end{array}$ & $0.7(0.6-0.9)^{*}$ & $<0.001$ & $0.7(0.6-0.8)^{*}$ & $<0.001$ & $1.0(0.8-1.3)$ & 0.816 & $0.9(0.8-0.9)^{*}$ & 0.001 & $0.8(0.7-0.8)^{*}$ & $<0.001$ & $1.1(1.0-1.3)$ & 0.090 \\
\hline - Hard physical activity & $1.3(1.1-1.5)^{*}$ & $<0.001$ & $0.9(0.8-1.1)$ & 0.547 & $1.4(1.1-1.8)^{*}$ & 0.011 & $1.1(1.0-1.2)$ & 0.084 & $1.1(0.9-1.3)$ & 0.288 & $1.0(0.8-1.2)$ & 0.985 \\
\hline $\begin{array}{l}\text { Global Health: } \\
\text { - Very good }\end{array}$ & Ref & & Ref & & & & Ref & & Ref & & & \\
\hline - Good & $1.3(1.1-1.6)^{*}$ & 0.009 & $1.4(1.1-1.8)^{*}$ & 0.002 & $0.9(0.7-1.2)$ & 0.570 & $1.4(1.2-1.6)^{*}$ & $<0.001$ & $1.5(1.3-1.8)^{*}$ & $<0.001$ & $0.9(0.7-1.1)$ & 0.340 \\
\hline - Fair & $2.0(1.6-2.6)^{*}$ & $<0.001$ & $2.4(1.8-3.1)^{*}$ & $<0.001$ & $0.8(0.6-1.2)$ & 0.379 & $1.8(1.5-2.1)^{*}$ & $<0.001$ & $2.5(2.1-3.0)^{*}$ & $<0.001$ & $0.7(0.5-0.9)^{*}$ & 0.003 \\
\hline - Poor & $2.9(1.9-4.5)^{*}$ & $<0.001$ & $3.8(2.5-5.9)^{*}$ & $<0.001$ & $0.8(0.4-1.4)$ & 0.382 & $2.5(1.8-3.4)^{*}$ & $<0.001$ & $3.5(2.5-4.9)^{*}$ & $<0.001$ & $0.7(0.4-1.1)$ & 0.139 \\
\hline $\begin{array}{l}\text { Anxiety and depression } \\
-0-4\end{array}$ & Ref & & Ref & & & & Ref & & Ref & & & \\
\hline$-5-9$ & $0.9(0.8-1.1)$ & 0.322 & $0.9(0.8-1.1)$ & 0.409 & $1.0(0.8-1.2)$ & 0.981 & $1.1(1.0-1.2)$ & 0.017 & $1.1(1.0-1.3)$ & 0.020 & $1.0(0.8-1.1)$ & 0.821 \\
\hline$-10-14$ & $1.0(0.8-1.1)$ & 0.619 & $1.1(0.9-1.3)$ & 0.511 & $0.9(0.7-1.2)$ & 0.412 & $1.2(1.1-1.4)^{*}$ & $<0.001$ & $1.3(1.1-1.5)^{*}$ & $<0.001$ & $0.9(0.8-1.1)$ & 0.482 \\
\hline$-15-19$ & $1.0(0.8-1.3)$ & 0.908 & $1.1(0.9-1.5)$ & 0.271 & $0.9(0.6-1.2)$ & 0.465 & $1.3(1.2-1.5)^{*}$ & $<0.001$ & $1.5(1.2-1.7)^{*}$ & $<0.001$ & $0.9(0.7-1.1)$ & 0.376 \\
\hline - 20 and higher & $0.8(0.6-1.1)$ & 0.198 & $1.2(0.9-1.6)$ & 0.279 & $0.7(0.4-1.1)$ & 0.093 & $1.1(0.9-1.4)$ & 0.215 & $1.3(1.1-1.6)^{*}$ & 0.009 & $0.9(0.6-1.1)$ & 0.295 \\
\hline Recent complaint & $1.9(1.5-2.3)^{*}$ & $<0.001$ & $0.9(0.8-1.1)$ & 0.267 & $2.0(1.6-2.6)^{*}$ & $<0.001$ & $1.8(1.5-2.1)^{*}$ & $<0.001$ & $1.0(0.9-1.1)$ & 0.787 & $1.8(1.4-2.1)^{*}$ & $<0.001$ \\
\hline Chronic complaint & $1.4(1.2-1.6)^{*}$ & $<0.001$ & $1.3(1.1-1.6)^{*}$ & $<0.001$ & $1.0(0.8-1.3)$ & 0.698 & $1.4(1.2-1.5)^{*}$ & $<0.001$ & $1.2(1.0-1.3)^{*}$ & 0.010 & $1.2(1.0-1.3)^{*}$ & 0.043 \\
\hline Psychiatric complaint & $1.3(1.1-1.5)$ & 0.011 & $1.9(1.6-2.3)^{*}$ & $<0.001$ & $0.7(0.5-0.9)^{*}$ & 0.002 & $1.2(1.0-1.3)^{*}$ & 0.005 & $1.4(1.2-1.5)^{*}$ & $<0.001$ & $0.8(0.7-1.0)^{*}$ & 0.032 \\
\hline
\end{tabular}


Table 3 Adjusted odds ratio (adjOR) with $95 \%$ confidence intervals $(95 \% \mathrm{Cl})$ from multivariate logistic regression models for visits to CAM practitioners for males in $2008(\mathrm{~N}=16311)$ and in $1997(\mathrm{~N}=16488)$, for females in $2008(\mathrm{~N}=20469)$ and in $1997(\mathrm{~N}=17587)$, and test of difference between the time points for males and females with Ratio Odds Ratio (ROR) (Continued)

\begin{tabular}{|c|c|c|c|c|c|c|c|c|c|c|c|c|}
\hline $\begin{array}{l}\text { Diseases } \\
\text { - Asthma }\end{array}$ & $0.8(0.7-1.0)$ & 0.048 & $0.9(0.8-1.2)$ & 0.501 & $0.9(0.6-1.2)$ & 0.368 & $1.0(0.9-1.1)$ & 0.834 & $1.1(0.9-1.2)$ & 0.431 & $0.9(0.8-1.1)$ & 0.462 \\
\hline - Hay fever & $1.0(0.9-1.2)$ & 0.706 & $1.3(1.1-1.6)^{*}$ & $<0.001$ & $0.8(0.6-1.0)^{*}$ & 0.015 & $1.1(1.0-1.2)$ & 0.017 & $1.3(1.2-1.4)^{*}$ & $<0.001$ & $0.9(0.7-1.0)^{*}$ & 0.019 \\
\hline - Heart disease & $0.7(0.6-0.9)^{*}$ & 0.002 & $0.7(0.5-0.9)^{*}$ & 0.001 & $1.1(0.8-1.5)$ & 0.496 & $0.9(0.7-1.0)$ & 0.072 & $0.9(0.7-1.1)$ & 0.366 & $1.0(0.7-1.3)$ & 0.805 \\
\hline - Cancer & $1.1(0.8-1.4)$ & 0.570 & $1.0(0.8-1.2)$ & 0.867 & $1.1(0.8-1.5)$ & 0.578 & $1.0(0.8-1.2)$ & 0.974 & $1.2(1.0-1.3)$ & 0.018 & $0.9(0.7-1.1)$ & 0.163 \\
\hline - Diabetes & $1.0(0.7-1.2)$ & 0.720 & $1.2(0.9-1.7)$ & 0.168 & $0.8(0.5-1.1)$ & 0.196 & $0.9(0.7-1.1)$ & 0.160 & $1.0(0.8-1.2)$ & 0.832 & $0.9(0.7-1.2)$ & 0.419 \\
\hline $\begin{array}{l}\text { - Musculoskeletal } \\
\text { disease }\end{array}$ & $1.1(1.0-1.3)$ & 0.118 & $0.8(0.5-1.1)$ & 0.175 & $1.5(1.0-2.2)$ & 0.065 & $1.1(1.0-1.2)$ & 0.033 & $0.8(0.6-1.1)$ & 0.168 & $1.4(1.0-1.9)$ & 0.050 \\
\hline $\begin{array}{l}\text { Visited last year: } \\
\text { - Physician }\end{array}$ & $1.8(1.5-2.2)^{*}$ & $<0.001$ & $2.0(1.7-2.4)^{*}$ & $<0.001$ & $0.9(0.7-1.1)$ & 0.342 & $1.6(1.4-1.9)^{*}$ & $<0.001$ & $1.9(1.7-2.1)^{*}$ & $<0.001$ & $0.9(0.7-1.0)$ & 0.119 \\
\hline - Chiropractor & $2.6(2.2-3.0)^{*}$ & $<0.001$ & $3.2(2.6-3.9)^{*}$ & $<0.001$ & $0.8(0.6-1.0)$ & 0.096 & $1.8(1.6-2.1)^{*}$ & $<0.001$ & $3.2(2.7-3.8)^{*}$ & $<0.001$ & $0.6(0.5-0.7)^{*}$ & $<0.001$ \\
\hline
\end{tabular}

A ROR above 1 indicates that the adjOR for 2008 is higher than for 1997.

* $\mathrm{p}$-value $<0.01$ for each year and $\mathrm{p}$-value $<0.05$ for comparison

a all variables are adjusted for all other variables in the model. 
There was a significant increase (ROR 2.0) in the odds of having a recent complaint in 2008 compared to 1997 , and having a recent complaint was associated with increased odds of CAM visits in 2008 (adjOR 1.9).

Having a university degree was associated with decreased odds of males visiting a CAM practitioner when comparing 2008 to 1997 (ROR 0.7) although not significantly associated with CAM visits in any year. In 1997 there was no association with marital status and CAM visits, while in 2008 Single males had decreased (adjOR 0.7) and widowers had increased (adjOR 1.7) odds of CAM visits and the only significant change from 1997 to 2008 was for widowers (ROR 1.8). Both in 1997 and 2008, being a smoker was associated with similar decreased odds of CAM visits (adjOR 0.7) while doing hard physical activity was associated with increased odds in 2008 (adjOR 1.3), a statistical significant increase from 1997 (ROR 1.4). Having a chronic complaint was associated with increased odds of CAM visits in both years (1997 adjOR 1.3, 2008 adjOR 1.4), while having psychiatric complaint was only associated with increased odds in 1997 (adjOR 1.9) with a reduction from 1997 to 2008 (ROR 0.7). In both 1997 and 2008 having a heart disease was associated with similar decreased odds of visits to CAM practitioners (adjOR 0.7), while having hay fever was associated with increased odds in 1997 (adjOR 1.3) but reduced between 1997 and 2008 (ROR 0.8).

\section{CAM visits females}

For females, having poorer self reported global health was associated with increased odds of CAM visits both in 1997 (adjOR from 1.5 for good health increasing to 3.5 for poor health) and in 2008 (adjOR from 1.4 for good health increasing to 2.5 for poor health), and although the odds was lower in 2008 it was only significantly different from 1997 for those with fair global health (ROR 0.7) (table 3). In 1997 being between 30 and 59 years was associated with increased odds (adjOR 1.3) while being above 80 years old was associated with decreased odds of visiting a CAM practitioner in 2008 (adjOR 0.8). There was a decrease from 1997 to 2008 for females aged 50 to 69 (ROR 0.8 to 0.7 ) and above 80 years (ROR 0.6). Visit to a physician (adjOR 1.9) or a chiropractor (adjOR 3.2) was also associated with CAM visits in 1997 and in 2008 (physician adjOR 1.6, chiropractor adjOR 1.8), with a significantly decrease from 1997 to 2008 for visits to chiropractor (ROR 0.6). Having a recent complaint was only associated with CAM visits in 2008 (adjOR 1.8), an increase from 1997 (ROR 1.8).

In 2008 (adjOR1.2), having middle level education was associated with increased odds of visiting a CAM practitioner. There was no association between marital status and CAM visits for females. Being a smoker was associated with similar decreased odds for CAM visits (1997
adjOR 0.8, 2008 adjOR 0.9). Increased anxiety and depression score (HADS-T) was associated with CAM visits both in 1997 (adj OR 1.1 for those with a score of 10-14 and above 20 to adj OR 1.5 for those with a score of 15-19) and 2008 (adj OR1.1 for those with a score of $10-14$ to 1.3 for those with a score of 15-19). Having a chronic complaint (adjOR 1.2 and 1.4) or psychiatric complaint (adjOR 1.4 and 1.2) was associated with increased odds of CAM visits in 1997 and 2008, with a reduction for psychiatric complaint from 1997 to 2008 (ROR 0.7) and an increase for chronic complaint (ROR 1.2). In 1997 (adjOR 1.3), having hay fever was associated with increased odds of CAM visits, a decrease from 1997 to 2008 (ROR 0.9).

\section{CAM visits in 2008; males vs. females}

There were most similarities among the characteristics of males and females CAM visitors in 2008 (table 4). Males had significantly $(\mathrm{p}<0.05)$ decreased odds of visiting a CAM practitioner compared to females if they were 50 years and older (ROR between 0.6 to 0.5 for those 50 to 79 years old), had university education (ROR 0.8 ), were single (ROR 0.7), had higher anxiety and depression score (HADS-T, from ROR 0.8 for those with a score of 5 to 14 to to ROR 0.6 for those with a score above 20) and hay fever (ROR 0.8). Widowers (ROR 1.6) and males with chronic complaint (ROR 1.2) had increased odds of visiting a CAM practitioner compared to females.

\section{Discussion}

From 1997 to 2008 there was a significant increase in the percentages of males and females visiting a CAM practitioner. For males, the significant changes were an increase in odds of visiting for those under 50 years, who had a recent complaint, were widower or did hard physical activities. There was a decrease for males who had a university degree, psychiatric complaint or hay fever. For females there was an increase in the odds for those under 50 years, who had a recent complaint or chronic complaint. It was a decrease for those with reported fair global health, psychiatric complaint, hay fever or if they had visited a chiropractor.

The main strength of this study is that it is the largest study to date comparing changes in characteristics of visitors to CAM practitioners. This allowed for both separate analysis for males and females and the analysis of a comprehensive set of explanatory variables. One major limitation was that it was only one question on CAM visits which prevents separate analysis based on frequency of visits. Furthermore, although the question mentioned several types of CAM practitioners, an even more comprehensive list would likely have increased the prevalence since it would enhance the respondents recall. The urban population was underrepresented in this study 
Table 4 Test of difference between males and females in 2008 with Ratio Odds Ratio (ROR)

\begin{tabular}{|c|c|c|}
\hline & \multicolumn{2}{|c|}{ Male vs. female 2008} \\
\hline & ROR $(95 \% \mathrm{Cl})$ & P-value 2) \\
\hline $\begin{array}{l}\text { Age group } \\
\text { - Under } 30\end{array}$ & Ref & \\
\hline$-30-39$ & $0.9(0.6-1.2)$ & 0.484 \\
\hline$-40-49$ & $0.8(0.6-1.1)$ & 0.202 \\
\hline$-50-59$ & $0.6(0.4-0.8)^{* *}$ & 0.001 \\
\hline$-60-69$ & $0.5(0.4-0.7)^{* *}$ & $<0.001$ \\
\hline$-70-79$ & $0.6(0.4-0.9)^{*}$ & 0.019 \\
\hline - Over 80 & $0.6(0.3-1.0)$ & 0.058 \\
\hline $\begin{array}{l}\text { Education } \\
\text { - Compulsory school }\end{array}$ & Ref & \\
\hline - Middle level & $0.9(0.7-1.1)$ & 0.225 \\
\hline - University & $0.8(0.6-1.0)^{*}$ & 0.038 \\
\hline $\begin{array}{l}\text { Marital status } \\
\text { - Married/cohabiting }\end{array}$ & Ref & \\
\hline - Single & $0.7(0.6-0.9)^{*}$ & 0.014 \\
\hline - Divorced/separated & $0.9(0.6-1.3)$ & 0.636 \\
\hline - Widow(er) & $1.6(1.0-2.5)^{*}$ & 0.032 \\
\hline Currently working & $0.9(0.7-1.1)$ & 0.353 \\
\hline $\begin{array}{l}\text { Current lifestyle: } \\
\text { - Daily smoker }\end{array}$ & $1.0(0.8-1.2)$ & 0.932 \\
\hline - Hard physical activity & $1.1(0.9-1.5)$ & 0.279 \\
\hline $\begin{array}{l}\text { Global Health: } \\
\text { - Very good }\end{array}$ & Ref & \\
\hline - Good & $1.0(0.8-1.3)$ & 0.814 \\
\hline - Fair & $0.9(0.7-1.3)$ & 0.664 \\
\hline - Poor & $1.0(0.5-1.8)$ & 0.940 \\
\hline $\begin{array}{l}\text { Anxiety and depression } \\
-0-4\end{array}$ & Ref & \\
\hline$-5-9$ & $0.8(0.7-1.0)^{*}$ & 0.024 \\
\hline$-10-14$ & $0.8(0.6-0.9)^{*}$ & 0.012 \\
\hline$-15-19$ & $0.7(0.5-1.0)^{*}$ & 0.024 \\
\hline - 20 and higher & $0.6(0.4-0.9)^{*}$ & 0.012 \\
\hline Recent complaint & $1.2(0.9-1.6)$ & 0.182 \\
\hline Chronic complaint & $1.2(1.0-1.5)^{*}$ & 0.048 \\
\hline Psychiatric complaint & $0.9(0.7-1.1)$ & 0.178 \\
\hline $\begin{array}{l}\text { Diseases } \\
\text { - Asthma }\end{array}$ & $0.8(0.6-1.0)$ & 0.080 \\
\hline - Hayfever & $0.8(0.7-1.0)^{*}$ & 0.030 \\
\hline - Heart disease & $0.9(0.6-1.2)$ & 0.394 \\
\hline - Cancer & $0.9(0.7-1.2)$ & 0.559 \\
\hline - Diabetes & $1.0(0.7-1.5)$ & 0.900 \\
\hline - Muscskelskeletal disease & $1.4(0.9-2.1)$ & 0.106 \\
\hline $\begin{array}{l}\text { Visited last year: } \\
\text { - Physician }\end{array}$ & $1.0(0.8-1.3)$ & 0.997 \\
\hline - Chiropractor & $0.8(0.7-1.1)$ & 0.172 \\
\hline
\end{tabular}

The test is based on the values given in table 2. A ROR above 1 indicates that the Adjusted OR for male is higher than for female.

* p-value $<0.05$

and a non-responder analysis of the 1997 data [18] found that older people were more likely not to answer the CAM question than younger people. Nevertheless, the age distribution was similar to other studies [6,19-21]. Chiropractors are authorised health personnel in Norway and were thus not included in the prevalence figures for CAM visits. This is likely to lower the prevalence compared to countries where chiropractors are CAM practitioners. Furthermore, CAM self care like use of products (herbs, vitamins) or practices (yoga) was not included and the prevalence is thus lower than for studies including such types of CAM. Studies which includes CAM self care [19], have similar profiles to this study.

The results may be affected by other factors that have not been recorded in both health surveys, i.e. the results may be affected by residual confounding. However, to our understanding, the main variables that lead to a visit to a CAM practitioner were included (general health, specific conditions, chronic conditions, socioeconomic situation). Importantly, the analyses were adjusted for having previously visited a general practitioner and chiropractor which in parts takes account of those who more frequently seek help as well as changes in health care utilisation between both surveys.

There was a substantial increase in the prevalence of CAM visits for both males and females during the 11 years, from $9.4 \%$ to $12.6 \%$. This coincides with a general increase in visits to physicians in the same time period, $17 \%$ points for males and $11 \%$ points for females (calculated from the numbers in table 2). The observed prevalence in this study is close to half of the CAM visits, which included chiropractors, in Australia [6], where there was an increase from $20.3 \%$ in 1993 to $26.5 \%$ in 2004. It was similar to a smaller Israeli study where the prevalence of CAM visitors also including chiropractors increased from $6.1 \%$ in 1993 via $9.8 \%$ in 2000 to $12.4 \%$ in 2007 [7]. Since the prevalence of visitors to CAM practitioners excluding chiropractors was similar in Norway and the USA in 2002 [5] and since the prevalence for practitioner based therapies like acupuncture has increased in USA [11], the prevalence seems to be is similar in the northern hemisphere but considerably lower than in Australia. However, in all countries there has been an increase in practitioner based CAM use during the last decade.

Consistent findings in studies of CAM use have been that middle aged people are the highest users. In this study, the age group among male CAM visitors increasing most was those under 30 years. Also for females the younger age groups had the largest increase. This trend is also observed in the USA were the middle aged do not longer stand out as clearly when 2007 [11] is compared to 2002 [19], a situation similar to Ireland [10]. This indicates some fundamental changes starting to happen in CAM consumption. The reduced influence of psychiatric complaints in this study could point in the same direction, and in the USA it is also observed that there is a 
reduction in how frequent psychological problems like anxiety and depression are named as a reason for CAM use $[11,19]$. Since having a recent complaint was among the variables with the largest increase from 1997 to 2008 this further strengthens the assumption. It indicates that a larger proportion of the more healthy part of the population is increasing their visits to CAM practitioners. Although this study can give no answers to why this is so, one speculation might be that children who have been taken to a CAM practitioner by their parents have continued to use CAM. This speculation builds on the observed fact that in Norway, there was an increase in the proportion of children among patients visiting homeopaths, from one in ten in 1985 to one in four in 1998 [22].

\section{Conclusions}

There has been an increase in the number of male and female adults who visit CAM practitioners from 1997 to 2008. The most prominent changes were that younger people of both genders with more limited complaints increased among the visitors.

\section{Acknowledgements}

Nord-Trøndelag Health Study (The HUNT Study) is a collaboration between HUNT Research Centre, Faculty of Medicine, Norwegian University of Science and Technology (NTNU, Verdal), The Norwegian Institute of Public Health, and Nord-Trøndelag County Council.

\section{Authors' contributions}

AS prepared the data file, performed the statistical analysis and drafted the manuscript. MBR and RJ helped to discuss the analysis and draft the manuscript. All authors have read and approved the final manuscript.

\section{Competing interests}

The authors declare that they have no competing interests.

Received: 25 January 2011 Accepted: 11 August 2011

Published: 11 August 2011

\section{References}

1. Bishop FL, Yardley L, Lewith GT: A systematic review of beliefs involved in the use of complementary and alternative medicine. $J$ Health Psychol 2007, 12(6):851-67.

2. Harris $P$, Rees $R$ : The prevalence of complementary and alternative medicine use among the general population: a systematic review of the literature. Complement Ther Med 2000, 8(2):88-96.

3. Quandt SA, Verhoef MJ, Arcury TA, Lewith GT, Steinsbekk A, Kristoffersen AE, Wahner-Roedler DL, Fonnebo V: Development of an international questionnaire to measure use of complementary and alternative medicine (I-CAM-Q). J Altern Complement Med 2009, 15(4):331-9.

4. Druss BG, Rosenheck RA: Use of practitioner-based complementary therapies by persons reporting mental conditions in the United States. Arch Gen Psychiatry 2000, 57(7):708-14.

5. Steinsbekk A, Rise MB, Aickin M: Cross-cultural comparison of visitors to CAM practitioners in the United States and Norway. J Altern Complement Med 2009, 15(11):1201-7.

6. MacLennan AH, Myers SP, Taylor AW: The continuing use of complementary and alternative medicine in South Australia: costs and beliefs in 2004. Med J Aust 2006, 184(1):27-31.

7. Shmueli A, Igudin I, Shuval J: Change and stability: use of complementary and alternative medicine in Israel: 1993, 2000 and 2007. Eur J Public Health 2010.
8. Wiles J, Rosenberg MW: 'Gentle caring experience'. Seeking alternative health care in Canada. Health Place 2001, 7(3):209-24.

9. Thomas $\mathrm{K}$, Coleman P: Use of complementary or alternative medicine in a general population in Great Britain. Results from the National Omnibus survey. J Public Health (Oxf) 2004, 26(2):152-7.

10. Fox $P$, Coughlan B, Butler $M$, Kelleher $C$ : Complementary alternative medicine (CAM. use in Ireland: a secondary analysis of SLAN data. Complement Ther Med 2010, 18(2):95-103.

11. Barnes PM, Bloom B, Nahin RL: Complementary and alternative medicine use among adults and children: United States, 2007. Natl Health Stat Report 2008, 12: 1-23.

12. Tindle HA, Davis RB, Phillips RS, Eisenberg DM: Trends in use of complementary and alternative medicine by US adults: 1997-2002. Altern Ther Health Med 2005, 11(1):42-9.

13. Eisenberg DM, Davis RB, Ettner SL, Appel S, Wilkey S, Van Rompay M, Kessler RC: Trends in alternative medicine use in the United States, 19901997: results of a follow-up national survey. JAMA 1998, 280(18):1569-75.

14. Sirois FM: Motivations for consulting complementary and alternative medicine practitioners: a comparison of consumers from 1997-8 and 2005. BMC Complement Altern Med 2008, 8:16.

15. Holmen J, Midthjell K, Krûger $\varnothing$, Langhammer A, Holmen T, Bratberg G, Vatten L, Lund-Larsen P: The Nord-Trøndelag Health Study 1995-97 (HUNT2): Objectives contents, methods and participation. Norsk Epidemiologi 2003, 13(1):19-32.

16. Harter M, Woll S, Wunsch A, Bengel J, Reuter K: Screening for mental disorders in cancer, cardiovascular and musculoskeletal diseases. Comparison of HADS and GHQ-12. Soc Psychiatry Psychiatr Epidemiol 2006, 41(1):56-62.

17. Altman $\mathrm{DG}$, Bland JM: Interaction revisited: the difference between two estimates. BMJ 2003, 326(7382):219.

18. Steinsbekk A, Adams J, Sibbritt D, Jacobsen G, Johnsen R: Sociodemographic characteristics and health perceptions among male and female visitors to CAM practitioners in a total population study. Forsch Komplementmed 2008, 15(3):146-51.

19. Barnes PM, Powell-Griner E, McFann K, Nahin RL: Complementary and alternative medicine use among adults: United States; 2002, , 343: 1-19, Adv Data 2004.

20. Menniti-Ippolito F, Gargiulo L, Bologna E, Forcella E, Raschetti R: Use of unconventional medicine in Italy: a nation-wide survey. Eur J Clin Pharmacol 2002, 58(1):61-4.

21. Hanssen B, Grimsgaard S, Launso L, Fonnebo V, Falkenberg T, Rasmussen NK: Use of complementary and alternative medicine in the Scandinavian countries. Scand J Prim Health Care 2005, 23(1):57-62.

22. Steinsbekk A, Fonnebo V: Users of homeopaths in Norway in 1998, compared to previous users and GP patients. Homeopathy 2003, 92(1):3-10.

Pre-publication history

The pre-publication history for this paper can be accessed here: http://www.biomedcentral.com/1472-6882/11/61/prepub

\section{doi:10.1186/1472-6882-11-61}

Cite this article as: Steinsbekk et al:: Changes among male and female visitors to practitioners of complementary and alternative medicine in a large adult Norwegian population from 1997 to 2008 (The HUNT studies). BMC Complementary and Alternative Medicine 2011 11:61. 